\title{
The frequency of virulent genes and antimicrobial resistance patterns of diarrheagenic Escherichia coli isolated from stools of children presenting with diarrhea in a tertiary hospital in Abakaliki, Nigeria
}

\author{
Ebuka Elijah David1(D), Muhammad Arfat Yameen², Ikechuku Okorie Igwenyi ${ }^{3}$, Arthur Chinedu Okafor ${ }^{4}$ (D) \\ Uket Nta Obeten ${ }^{1}$, Doris Olachi Obasi ${ }^{1}$, Uchenna Regina Ezeilo ${ }^{1}$ iD and Chidinma Nnenne David ${ }^{5}$
}

1. Department of Chemistry/Biochemistry, Alex-Ekwueme Federal University Ndufu-Alike, Ikwo, Ebonyi State, Nigeria;

2. Department of Pharmacy, COMSATS Institute of Information Technology, Abbottabad, Pakistan; 3. Department of

Biochemistry, Ebonyi State University, Ebonyi State, Nigeria; 4. Department of Microbiology, Edo University, Iyamho, Edo State, Nigeria; 5. Department of Microbiology, Ebonyi State University, Ebonyi State, Nigeria.

Corresponding author: Ebuka Elijah David, e-mail: david.ebuka@funai.edu.ng

Co-authors: MAY: arfatyameen@gmail.com, IOI: igwenyike@yahoo.com, ACO: arthur.okafor@edouniversity.edu.ng, UNO: uketgabriel@gmail.com, DOO: obasidoris19@gmail.com, URE: ucmgbenka@gmail.com, CND: nnenne.nnaji@gmail.com

Received: 21-06-2020, Accepted: 12-08-2020, Published online: 01-10-2020

doi: www.doi.org/10.14202/IJOH.2020.147-152 How to cite this article: David EE, Yameen MA, Igwenyi IO, Okafor AC, Obeten UN, Obasi DO, Ezeilo UR, David CN (2020) The frequency of virulent genes and antimicrobial resistance patterns of diarrheagenic Escherichia coli isolated from stools of children presenting with diarrhea in a tertiary hospital in Abakaliki, Nigeria, Int. J. One Health, 6(2): 147-152.

\begin{abstract}
Aim: This study was aimed to determine the virulent genes and antibiotic resistance patterns among circulating diarrheagenic Escherichia coli (DEC) pathotypes in a tertiary care health center in east of Nigeria.

Materials and Methods: Diarrheal stool samples were obtained from 80 children under 5 years and E. coli was isolated and identified using standard biochemical and molecular methods. Multiplex polymerase chain reaction (PCR) was used to detect eight virulent genes of DEC. Disk diffusion method was used to determine the antibiotic susceptibility of DEC.

Results: DEC infection was observed in 54 (68\%) children among which ial gene for enteroinvasive E. coli (EIEC) $(40 \%$ [ $\mathrm{n}=22])$ was commonly detected followed by eltA/eltB for enterotoxigenic E. coli (ETEC) $(30 \%$ [ $\mathrm{n}=16]), p C V D$ for enteroaggregative E. coli (EAEC) $(20 \%$ [n=11]), and eaeA/bfpA for typical enteropathogenic $E$. coli (EPEC) $(10 \%$ [n=5]). The DEC isolates phenotypically exhibited resistance for ampicillin (AMP) (44 [81\%]), followed by ciprofloxacin (CIP)/ levofloxacin (LEV) (28 [52\%]), cefoxitin (FOX) (11 [20\%]), and amoxicillin-clavulanic acid (AMC) (6 [11\%]). About 60\% isolates of stable toxins-ETEC were resistant to AMC, CIP, and LEV while all the labile toxin-ETEC exhibited resistance to AMP. About $60 \%(\mathrm{n}=6)$ resistance were seen in EAEC against ampicillin, AMC, FOX, CIP, and LEV. In EIEC, all the isolates $(\mathrm{n}=22)$ were resistant to AMP while $50 \%(\mathrm{n}=11)$ were resistant to both CIP and LEV. All EPEC $(\mathrm{n}=5)$ were resistant to AMP, FOX, CIP, and LEV.
\end{abstract}

Conclusion: High frequency of virulent ial and eltA/eltB genes for EIEC and ETEC, respectively, suggests that they are the primary etiological agents of diarrhea in children among DEC pathotypes. Resistance of DEC to more than two classes of antibiotics indicate possible emergence of multidrug resistance.

Keywords: antibiotic resistance, diarrheagenic Escherichia coli, diarrheal stool, multidrug resistance, multiplex polymerase chain reaction, polymerase chain reaction.

\section{Introduction}

Escherichia coli are Gram-negative, oxidase-negative, rod-shaped bacteria from the family Enterobacteriaceae [1]. E. coli are commensal bacteria found in the intestinal microflora of a variety of animals, including man. All the strains of $E$. coli are not harmless since some can cause debilitating and sometimes fatal diseases in humans and birds [2]. Pathogenic strains of E. coli are divided into intestinal pathogenic E. coli (InPEC) causing diarrhea and

Copyright: David, et al. This article is an open access article distributed under the terms of the Creative Commons Attribution 4.0 International License (http://creativecommons.org/licenses/ by/4.0/), which permits unrestricted use, distribution, and reproduction in any medium, provided you give appropriate credit to the original author(s) and the source, provide a link to the Creative Commons license, and indicate if changes were made. The Creative Commons Public Domain Dedication waiver (http:// creativecommons.org/ publicdomain/zero/1.0/) applies to the data made available in this article, unless otherwise stated. extraintestinal pathogenic E. coli (ExPEC) causing a variety of infections, including urinary tract infections, meningitis, and septicemia [3,4]. InPEC strains of $E$. coli, also known as diarrheagenic E. coli (DEC), are a major etiological agent of pediatric diarrhea, accounting for over 2 million deaths annually [5]. DEC continues to be the most common cause of infantile morbidity and mortality, most especially in developing countries and sub-Saharan Africa [6], especially in children under 5 years of age [7]. Nigeria reported at least $20.6 \%$ diarrhea-specific deaths, representing about 103,000 of the 500,000 annual global pediatric deaths [1]. DEC can be transmitted through the fecal-oral route by ingesting food or water contaminated by human or animal feces [6,8]. Infection with DEC causes an alteration of the movement of ions and water in the gastrointestinal tract by altering the balance between fluid-electrolyte absorption 
and secretion leading to diarrhea [9]. DEC is divided into enteropathogenic $E$. coli (EPEC), enterotoxigenic $E$. coli (ETEC), enterohemorrhagic E. coli, enteroinvasive E. coli (EIEC), enteroaggregative E. coli (EAEC), and diffusely adherent $E$. coli. The divisions of DEC into groups are based on their specific virulence factors and phenotypic traits. Each pathological type has characteristic virulence determinants that contribute to its pathogenic mechanisms [10]. The prevalence and other epidemiological features of DEC types in childhood diarrhea vary with geographical area [5].

Recently, there is an emergence of multidrug resistance in all pathological types of $E$. coli isolated from children with diarrhea, which makes treatment challenging [11]. In the last decade, resistance against the commonly used drugs for the treatment of enteric infections, including ampicillin (AMP), tetracycline, and cotrimoxazole has increased among DEC [12], which have led to the use of higher antimicrobials like fluoroquinolones as alternatives. In addition, the use and misuse of these empirical drugs in these informal sectors, by unlicensed providers and through self-medication, are commonplace in Nigeria and in many other parts of Africa, which has led to both single drug and multidrug resistance [13]. The frequency of DEC and its multidrug resistance in childhood diarrhea has been reported in provincial Southwest and North Central Nigeria [14,15], but little or no information is available for Southeastern part of Nigeria.

The aim of this study was to investigate the frequency of virulent genes and antimicrobial resistance patterns of DEC isolated from stools of children presenting with diarrhea in Alex Ekwueme Federal Teaching Hospital, Abakaliki (AE-FETHA), Ebonyi State, Nigeria.

\section{Materials and Methods}

\section{Ethical approval and informed consent}

Ethical clearance (Ref. No.: FETHA/REC/ VOL.1/2016/386) was obtained from Ethical and Research Committee of the hospital, after which informed consent was obtained from the parents/ guardians/attendants of the children.

\section{Isolation and identification of $E$. coli}

Eighty fecal samples were collected from children with incidence of diarrhea under the age of 5 years at AE-FETHA from August to October 2018. Fecal samples were processed according to the standard guidelines provided for laboratory diagnosis of enteric pathogens [16]. A loop full of samples was suspended in sterile nutrient broth and incubated at $37^{\circ} \mathrm{C}$ for $24 \mathrm{~h}$. The isolates from the broth were inoculated directly on MacConkey's agar plates. The isolates were picked up after overnight incubation at $37^{\circ} \mathrm{C}$ and subcultured in Eosin Methylene Blue, EMB (a selective media for E. coli) agar plates at $37^{\circ} \mathrm{C}$ for $24 \mathrm{~h}$. Other biochemical tests such as indole test, methyl red test, Voges-Proskauer test, citrate utilization test, and
Eijkman test were performed on colonies that showed green metallic sheen, typical of $E$. coli isolates.

Standard cetyltrimethylammonium-bromide/ sodium chloride method was used to extract E. coli genomic DNA, as previously described by Healey et al. [17]. The DNA concentrations were measured in $n g / \mu \mathrm{L}$ using Nanodrop instrument (Colibri Spectrometer, Berthold Detection System, Germany). The quality of DNA was analyzed by electrophoresis in $1 \%$ agarose gels in TBE buffer at $100 \mathrm{~V}$. The gels were stained with ethidium bromide and photographed under ultraviolet light using a gel documentation system. E. coli isolates were further identified using primers derived from the DNA sequences flanking the gene encoding the universal stress protein $\mathrm{A}$ (uspA);

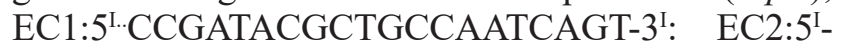
ACGCAGACCGTAAGGGCCAGAT-3 ${ }^{\mathrm{I}} \quad[18,19]$. Polymerase chain reaction (PCR) was performed in a total reaction volume of $25 \mu \mathrm{L}$ with $12.5 \mu \mathrm{L}$ GoTaq Green Master Mix, 9.0 $\mu \mathrm{L}$ nuclease-free water (Promega, USA), $0.5 \mu \mathrm{L}$ of forward and reverse primers each, and $2.5 \mu \mathrm{L}$ of template. Optimization was done at the following conditions: $94^{\circ}$ for $5 \mathrm{~min}$, initial template denaturation, 25 cycles at $94^{\circ}$ for $30 \mathrm{~s}$, final denaturation, $50^{\circ}$ for $1 \mathrm{~min}$, annealing, $72^{\circ}$ for $1 \mathrm{~min}$ $30 \mathrm{~s}$, extension, and $72^{\circ} 7 \mathrm{~min}$, final extension. About 884 bp PCR products were analyzed by gel electrophoresis in $1 \%$ agarose gel in TBE buffer at $100 \mathrm{~V}$. The gels were stained with ethidium bromide and photographed under ultraviolet light using a gel documentation system (Figure-S1 in Additional file-1).

\section{Detection of virulent genes in DEC}

Detection of virulent genes of DEC was performed in a group of multiplex PCR using their specific primers as previously described by Nguyen et al. [20]. All the primers and their corresponding virulent genes are shown in Table-S1 (Additional file-1). The thermocycling conditions were programmed using Applied Biosystem, 2720 Thermal Cycler, USA, in $25 \mu \mathrm{L}$ reaction mixture as follows: Initial denaturation for $5 \mathrm{~min}$ at $94^{\circ} \mathrm{C}$, denaturation at $94^{\circ} \mathrm{C}$ for $30 \mathrm{~s}$, $72^{\circ} \mathrm{C}$ for $1 \mathrm{~min} 30 \mathrm{~s}$, and extensions for 25 cycles with final extension of $5 \mathrm{~min}$ at $72^{\circ} \mathrm{C}$. PCR products were analyzed by gel electrophoresis in $1 \%$ agarose gel in TBE buffer at $100 \mathrm{~V}$. The gels were stained with ethidium bromide and photographed under ultraviolet light using a gel documentation system (Figure-S2 in Additional file-1).

\section{Antimicrobial susceptibility testing}

Antimicrobial susceptibility testing was performed by the disk diffusion method according to the European Committee on Antimicrobial Susceptibility Testing [21] guidelines for Enterobacteriaceae. Antimicrobial agents tested were AMP $(10 \mu \mathrm{g})$, amoxicillin/clavulanic acid (AMC) $(5 \mu \mathrm{g})$, cefoxitin (FOX) $(30 \mu \mathrm{g})$, ciprofloxacin (CIP) $(5 \mu \mathrm{g})$, imipenem (IMI) $(10 \mu \mathrm{g})$, and levofloxacin (LEV) $(5 \mu \mathrm{g})$ (Oxoid Ltd., Basingstoke, Hampshire, England). 
The multidrug resistance criteria adopted were defined as earlier published by Magiorakos et al. [22]. E. coli ATCC 25922 was used as quality control.

\section{Results}

\section{Frequency of DEC virulent pathotypes}

A total of 80 diarrheal stool samples from diarrheal children $(\mathrm{n}=80)$ were collected for this study. Of the 80 children, $68 \%(n=54)$ were found to be positive for one or more pathotype of DEC. Virulent eltA gene for stable toxins-ETEC (ST-ETEC) was detected in $20 \%(n=11)$ of the children while $10 \%(n=5)$ expressed eltB for labile toxin-ETEC (LT-ETEC). About 20\% $(\mathrm{n}=11)$ also expressed $\mathrm{pCVD}$ (the nucleotide sequence of EcoR1-Pst1 DNA fragment of pCVD432 of EAEC) gene for EAEC while $40 \%(\mathrm{n}=22)$ expressed ial gene (invasion-associated locus of the invasive plasmid found in EIEC). About 10\% $(\mathrm{n}=5)$ expressed eaeA gene (a structural gene for intimin) and $b f p A$ gene (a structural gene for the bundle-forming pilus) found in typical EPEC, Figure-1.

\section{Antibiotic susceptibility testing}

The DEC isolates phenotypically exhibited resistance for AMP (44 [81\%]) followed by CIP and LEV (28 [52\%]), FOX (11 [20\%]) and AMC (6 [11\%]). None of the isolates were resistant to IMI $(0[0 \%])$, Table- 1 .

About $60 \%$ isolates of ST-ETEC were resistant to AMC, CIP, and LEV while all the LT-ETEC exhibited resistance to AMP. About $60 \%$ of resistance were seen in EAEC against ampicillin, AMC, FOX, CIP, and LEV. In EIEC, all the isolates were resistant to AMP while 50\% were resistant to both CIP and LEV. All EPEC were resistant to AMP, FOX, CIP, and LEV, Table-2. Multidrug resistance was seen in greater number of all the pathotypes except LT-ETEC.

\section{Discussion}

In this study, the frequency of EIEC (40\% [22/54]) was most common among DEC, followed by ETEC (30\% [16/54]), EAEC (20\% [11/54]), and EPEC (10\% [5/54]). The high frequency of EIEC and ETEC among DEC pathotypes suggests their role as most common cause of acute childhood diarrhea in this region. In contrast to high EIEC frequency recorded in this study, the low rate $(1.2 \%)$ of recovery of EIEC in the study carried out in Southwest Nigeria by Okeke et al. [14], in India (1.8\%) [23], and Ecuador (3.2\%) [24], suggested that EIEC may play less important role in childhood diarrhea in developing countries. EIEC infection is characterized by the ability of bacteria to invade the human colonic mucosa, conferred by the expression of chromosomal and plasmid-borne genes [3]. High frequencies of ETEC have been recorded in other parts of Nigeria [25] and other developing countries [26]. The high ETEC frequency from this study also agreed with the one recorded in Onitsha, Southeast Nigeria, where $21.57 \%$ ETEC strains were detected among DEC isolates [25]. Similar high ETEC frequency (36.3\%) among hospitalized diarrheal children in Kolkata, India,

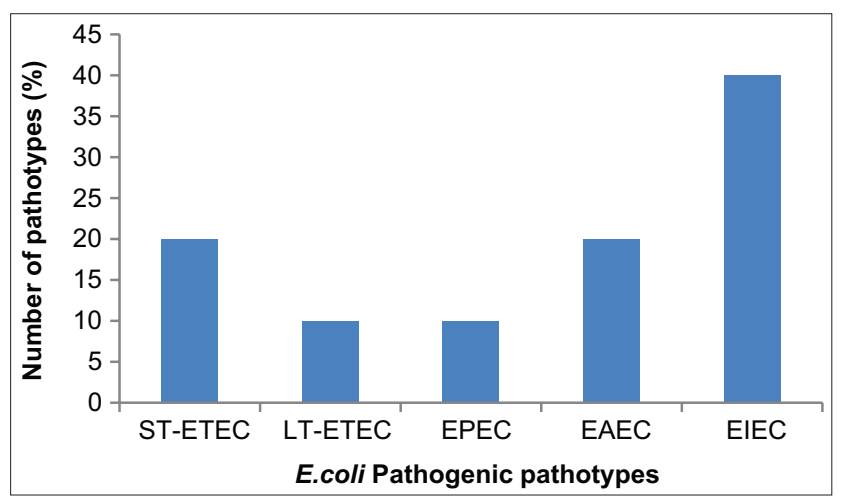

Figure-1: Distribution of diarrheagenic Escherichia coli pathotypes.

Table-1: Antibiogram of diarrheagenic Escherichia coli isolates $(n=54)$.

\begin{tabular}{lcc}
\hline Antimicrobials & Resistant (\%) & Sensitivity (\%) \\
\hline AMP & $44(81)$ & $10(19)$ \\
AMC & $6(11)$ & $48(89)$ \\
FOX & $11(20)$ & $43(80)$ \\
IMP & $0(0)$ & $54(100)$ \\
CIP & $28(52)$ & $26(48)$ \\
LEV & $28(52)$ & $26(48)$ \\
\hline
\end{tabular}

$\mathrm{AMP}=$ Ampicillin, $\mathrm{AMC}=$ Amoxicillin-clavulanic acid, FOX =Cefoxitin, IMP=Imipenem, CIP=Ciprofloxacin, LEV $=$ Levofloxacin

was also recorded [26]. Our result was different from the frequency of ETEC obtained among DEC isolated from infants and children in Federal Capital Territory, Abuja, Nigeria, where 4\% ETEC frequency was recorded [27] and other developing countries; Dar es Salaam, Tanzania (3.6\%) [28]; West Iran (17.5\%) [29]; and Hanoi, Vietnam (2.2\%) [20]. Consistent with our result, ETEC was not the most prevalent. Clinical human ETEC isolates produce enterotoxins; the heat ST and heat LT and may produce one or more of several colonization factors which mediate adherence to the small intestinal mucosa [30]. In this study, the heat stable ETEC, ST-ETEC showed greater association with childhood diarrhea than heat labile, LT-ETEC. This result agreed with the one performed in Southwest Nigeria [14] and other parts of the world [31,32].

In this study, EAEC ranked second to typical EPEC as the least frequent DEC. When compared to results obtained from other regions, it becomes evident that the prevalence and other epidemiological features of DEC types in childhood diarrhea vary with geographical area [5]. Similar to our result, EPEC was the least prevalent in Ecuador (0.9\%) [24], India 4.79\% [23], and Egypt (5.2\%) [33]. Different from the result of this study, EAEC was recorded as the most prevalent in Kolkata, India (48.2\%) [26], Southwest Nigeria (10.3\%) [14], and $34.4 \%$ in Gwagwalada, Abuja, Nigeria [15].

The DEC isolates were most resistant to AMP, followed by CIP/LEV, FOX, and AMC. Most worrisome is that more than $50 \%$ of all the DEC was multidrug and fluoroquinolone (CIP/LEV) resistant. This result is similar with a previous study where most of the DEC 
Table-2: Relationship between diarrheagenic Escherichia coli virulent pathotypes and antimicrobial resistance.

\begin{tabular}{|c|c|c|c|c|c|c|c|c|c|}
\hline DEC & $n$ & Vir. gene & AMP & AMC & FOX & CIP & LEV & IMP & NODR \\
\hline ST-ETEC & 11 & eltA & $60 \%(6)$ & $0 \%(0)$ & $0 \%(0)$ & $60 \%(6)$ & $60 \%(6)$ & $0 \%(0)$ & 3 \\
\hline LT-ETEC & 05 & eltB & $100 \%(5)$ & $0 \%(0)$ & $0 \%(0)$ & $0 \%(0)$ & $0 \%(0)$ & $0 \%(0)$ & 1 \\
\hline EAEC & 11 & $p C V D$ & $60 \%(6)$ & $60 \%(6)$ & $60 \%(6)$ & $60 \%(6)$ & $60 \%(6)$ & $0 \%(0)$ & 5 \\
\hline EIEC & 22 & ial & $100 \%(22)$ & $0 \%(0)$ & $0 \%(0)$ & $50 \%(11)$ & $50 \%(11)$ & $0 \%(0)$ & 3 \\
\hline EPEC & 05 & eae $A$ and $b f p A$ & $100 \%(5)$ & $0 \%(0)$ & $100 \%(5)$ & $100 \%(5)$ & $100 \%(5)$ & $0 \%(0)$ & 4 \\
\hline
\end{tabular}

$\mathrm{AMP}=$ Ampicillin, $\mathrm{AMC}=$ Amoxicillin-clavulanic acid, FOX=Cefoxitin, IMP=Imipenem, CIP=Ciprofloxacin, LEV=Levofloxacin, NODR $=$ number of drug resistance

isolates $(67.5 \%)$ were resistant to AMP and tetracycline with ETEC being significantly more resistant to CIP than other DEC group [29]. Such multidrug resistance among DEC isolates against classical antibiotics such as AMP and tetracycline was also recorded in Bolivia, although no fluoroquinolone resistance was observed [30]. Correlation between antimicrobial resistant pattern of $E$. coli from environmental water and clinical diarrheal stool samples in Lagos, Nigeria, further supported our findings that most of the isolates ( $82 \%$ ) were resistant to multidrug, including fluoroquinolones [34]. The fluoroquinolone resistance in E. coli has been commonly associated with mutations in the gyrA, subunit A of gyrase in Gram-negatives [35]. The use of fluoroquinolones as first drug of choice for diarrhea in developing countries where the use of antibiotics is not regulated may lead to a rapid emergence of resistance.. Much of the reasons for these high rates of resistance are related to the fact that, antibiotics, despite not being required for the treatment of acute diarrhea, are widely prescribed for these forms of infections [36]. There is also the difficulty in understanding the complicated dynamics of antimicrobial resistance transmission between humans and animals sharing the same environment. Hence, the need to apply a One Health approach and study environmental reservoirs more closely, rather than focusing only on the resistance that arises following antimicrobial administration [37].

\section{Conclusion}

We observed the presence of four different DEC pathotypes with EIEC and ETEC most commonly encountered. High frequency of EIEC and ETEC in this region suggests that it is one of the most common causes of diarrhea in children below 5 years. Most of these DEC are resistant to more than 1 antimicrobial agent. The availability of over-the-counter antibiotics and continued use and misuse of these drugs allow for the selection of resistant isolates. Education of the parents/guardians of these children on the management of diarrheal infections as well as the implementation of more stringent policies governing the empirical treatment of diarrhea by physicians is advised. Therefore, it is important to continue the surveillance of antimicrobial resistance of enteric bacterial pathogens for effective control of childhood diarrheal diseases.

\section{Authors' Contributions}

EED: Conceptualization and writing. MAY: Microbial identification and molecular analysis. IOI: Review and final editing. ACO and UNO: Sample collection and microbial culture. DOO and URE: Interpretation and data analysis. CND: Coordination and formatting. All authors read and approved the final manuscript.

\section{Acknowledgments}

The authors are very grateful for the financial support received from The World Academy of Sciences in collaboration with Higher Education Commission, Pakistan, through COMSATS Institute of Information Technology, Abbottabad, Pakistan (FR number: 3240287158). The funding body had no role in either the design of the study, collection of samples, analysis, and interpretation of data or in writing/publishing of the manuscript.

\section{Competing Interests}

The authors declare that they have no competing interests.

\section{Publisher's Note}

Veterinary World (Publisher of International Journal of One Health) remains neutral with regard to jurisdictional claims in published institutional affiliation.

\section{References}

1. Croxen, M.A., Law, R.J., Scholz, R., Keeney, K.M., Wlodarska, M. and Finlay, B.B. (2013) Recent advances in understanding enteric pathogenic Escherichia coli. Clin. Microbiol. Rev., 26(4): 822-880.

2. Bélanger, L., Garenaux, A., Harel, J., Boulianne, M., Nadeau, E. and Dozois, C.M. (2011) Escherichia coli from animal reservoirs as a potential source of human extraintestinal pathogenic E. coli. Pathog. Dis., 62(1): 1-10.

3. Paterson, D.L. and Doi, Y. (2017) Enterobacteriaceae. In: Mayers, D., Sobel, J., Ouellette, M., Kaye, K. and Marchaim, D., editors. Antimicrobial Drug Resistance. Springer, Cham.

4. LeStrange, K., Markland, S.M., Hoover, D.G., Sharma, M. and Kniel, K.E. (2017) An evaluation of the virulence and adherence properties of avian pathogenic Escherichia coli. One Health, 4: 22-26.

5. Verma, S., Venkatesh, V., Kumar, R., Kashyap, S., Kumar, M., Maurya, A.K., Dhole, T.N. and Singh, M. (2019) Etiological agents of diarrhea in hospitalized pediatric patients with special emphasis on diarrheagenic Escherichia coli in North India. J. Lab. Physicians, 11(1): 68-74.

6. Adefisoye, M.A. and Okoh, A.I. (2016). Identification and antimicrobial resistance prevalence of pathogenic Escherichia coli strain from treated wastewater effluents in Eastern Cape, South Africa. Open. Microbiol. J., 5(1): 143-151.

7. World Health Organization. (2012) World Health Statistics: A Snapshot of Global Health. World Health Organization, 
Geneva.

8. Udedi, S.C., David, E.E., Igwilo, I.O., Ekwealor, K.E., Enemali, M.O., Bamidele T.O., Ifemeje, J.C. and Asogwa, K.K. (2013) Antidiarrhoeal activity of cashew (Anacardium occidentale) leaf extract enriched with zinc in Wistar albino rats. Sky J. Biochem. Res., 2(6): 37-41.

9. Soleimani, A., Foroozanfard, F. and Tamadon, M.R. (2017) Evaluation of water and electrolytes disorders in severe acute diarrhea patients treated by WHO protocol in eight large hospitals in Tehran; a nephrology viewpoint. J. Rennal Inj. Prev., 6(2): 109-112.

10. Sarowska, J., Futoma-Koloch, B., Jama-Kmiecik, A., FrejMadrzak, M., Ksiazczyk, M., Bugla-Ploskonska, G. and Choroszy-Krol, I. (2019) Virulence factors, prevalence and potential transmission of extraintestinal pathogenic Escherichia coli isolated from different sources: Recent reports. Gut Pathog., 11(1): 10.

11. Klemm, E.J., Wong, V.K. and Dougan, G. (2018) Emergence of dominant multidrug-resistant bacterial clades: Lessons from history and whole-genome sequencing. Proc. Natl. Acad. Sci U. S. A., 115(51): 12872-12877.

12. DuPont, H.L. and Steffen, R. (2017) Use of antimicrobial agents for treatment and prevention of travellers' diarrhoea in the face of enhanced risk of transient fecal carriage of multidrug resistant Enterobacteriaceae: Setting the stage for consensus recommendations. J. Travel. Med., 24(1): S57-S62.

13. Binali, D., Musaya, J. and Lampiao, F. (2018) Determinants of antimicrobial use in diarrhoea management among underfive children in Zomba, Malawi. J. Microbiol. Antimicrob., 11(2): 22-30.

14. Okeke, I.N., Lamikanra, A., Steinrück, H. and Kaper, J.B. (2000) Characterization of Escherichia coli strains from cases of childhood diarrhea in provincial Southwestern Nigeria. J. Clin. Microbiol., 38(1): 7-12.

15. Onanuga, A., Igbeneghu, O. and Lamikanra, A. (2014) A study of the prevalence of diarrhoeagenic Escherichia coli in children from Gwagwalada, Federal Capital Territory, Nigeria. Pan Afr. Med. J., 17: 146.

16. Cheesbrough, M. (2006) District Laboratory Practice In Tropical Countries. Cambridge University Press, Cambridge, England.

17. Healey, A., Furtado, A., Cooper, T. and Henry, R.J. (2014) Protocol: A simple method for extracting next-generation sequencing quality genomic DNA from recalcitrant plant species. Plant Methods, 10(1): 21.

18. Anil, K.M., Deepak, S.D., Gururaj, K., Geetika, G., Nitika, S., Naveen, K., Shivasharanappa, N. and Souvik, P. (2017) UspA gene based characterization of Escherichia coli strains isolated from different disease conditions in goats. J. Anim. Res., 7(6): 1123-1128.

19. Yameen, M.A., David, E.E., Nzelibe, H.C., Shuaibu, M.N., Magaji, R.A., Ogugu, A.J. and Onwe, O.S. (2018) Molecular characterization of enterotoxigenic Escherichia coli: Effect on intestinal nitric oxide in diarrheal disease. J. Bacteriol. Parasitol., 9: 339.

20. Nguyen, T.V., Le Van, P., Le Huy, C., Gia, K.N. and Weintraub, A. (2005) Detection and characterization of diarrheagenic Escherichia coli from young children in Hanoi, Vietnam. J. Clin. Microbiol., 43(2): 755-760.

21. The European Committee on Antimicrobial Susceptibility Testing. (2019) Breakpoint Tables for Interpretation of MICs and Zone Diameters. Version 6.0, 2019. European Committee on Antimicrobial Susceptibility Testing, Växjö, Sweden. Available from: http://www.eucast.org. Retrieved on 20-02-2020.

22. Magiorakos, A.P., Srinivasan, A., Carey, R., Carmeli, Y., Falagas, M., Giske, C., Harbarth, S., Hindler, J., Kahlmeter, G. and Olsson-Liljequist, B. (2012) Multidrugresistant, extensively drug-resistant and pandrug-resistant bacteria: An international expert proposal for interim standard definitions for acquired resistance. Clin. Microbiol. Infect., 18(3): 268-281.

23. Chellapandi, K., Dutta, T.K., Sharma, I., Sharma, I., de Mandal, S., Kumar, N.S. and Ralte, L. (2017) Prevalence of multi drug-resistant enteropathogenic and enteroinvasive Escherichia coli isolated from children with and without diarrhea in Northeast Indian population. Ann. Clin. Microb. Antimicrob, 16(1): 49.

24. Vieira, N., Bates, S.J., Solberg, O.W., Ponce, K., Howsmon, R., Cevallos, W., Trueba, G., Riley, L. and Eisenberg, J.N.S. (2008) High prevalence of enteroinvasive Escherichia coli isolated in a remote region of Northern coastal Ecuador. Am. J. Trop. Med. Hyg., 76(3): 528-533.

25. Nweze, E. (2010) Aetiology of diarrhoea and virulence properties of diarrhoeagenic Escherichia coli among patients and healthy subjects in Southeast Nigeria. J. Health Popul. Nutr, 28(3): 245.

26. Dutta, S., Guin, S., Ghosh, S., Pazhani, G.P., Rajendran, K., Bhattacharya, M.K., Takeda, Y., Nair, G.B. and Ramamurthy, T. (2013) Trends in the prevalence of diarrheagenic Escherichia coli among hospitalized diarrheal patients in Kolkata, India. PLoS One, 8(2): e56068.

27. Ifeanyi, C.I.C., Ikeneche, N.F., Bassey, B.E., Al-Gallas, N., Aissa, R.B. and Boudabous, A. (2015) Diarrheagenic Escherichia coli pathotypes isolated from children with diarrhea in the federal capital territory Abuja, Nigeria. $J$. Infecet. Dev. Ctries., 9(2): 165-174.

28. Moyo, S.J., Maselle, S.Y., Matee, M.I., Langeland, N. and Mylvaganam, H. (2007) Identification of diarrheagenic Escherichia coli isolated from infants and children in dar es Salaam, Tanzania. BMC Infect. Dis., 7(1): 92.

29. Alikhani, M.Y., Hashemi, S.H., Aslani, M.M. and Farajnia, S. (2013) Prevalence and antibiotic resistance patterns of diarrheagenic Escherichia coli isolated from adolescents and adults in Hamedan, Western Iran. Iran. $J$. Microbiol., 5(1): 42.

30. Rodas, C., Mamani, R., Blanco, J., Blanco, J.E., Wiklund, G., Svennerholm, A.M., Sjöling, A. and Iniguez,V. (2011) Enterotoxins, colonization factors, serotypes and antimicrobial resistance of enterotoxigenic Escherichia coli (etec) strains isolated from hospitalized children with diarrhea in Bolivia. Braz. J. Infect. Dis., 15(2): 132-137.

31. Gunzburg, S., Chang, B., Burke, V. and Gracey, M. (1992) Virulence factors of enteric Escherichia coli in young aboriginal children in North-West Australia. Epidemiol. Infecet., 109(2): 283-289.

32. Porat, N., Levy, A., Fraser, D., Deckelbaum, R.J. and Dagan, R. (1998) Prevalence of intestinal infections caused by diarrheagenic Escherichia coli in bedouin infants and young children in Southern Israel. Pediatr. Infect. Dis. J., 17(6): 482-488.

33. Behiry, I.K., Abada, E.A., Ahmed, E.A. and Labeeb, R.S. (2011) Enteropathogenic Escherichia coli associated with diarrhea in children in Cairo, Egypt. Sci. World J., 11: 2613-2619.

34. Azenabor, A., Igbokwe, H.N., Bhattacharyya, S., Gradus, S.M., Khubbar, M., Griswold, D., Navidad, J. and Igwilo, C. (2013) Multidrug resistance pattern in Escherichia coli: The evolving threat of antimicrobial resistance in a developing country. Am. J. Clin. Pathol., 140(Suppl 1): A045-A045.

35. Vila, J., Vargas, M., Ruiz, J., Corachan, M., De Anta, M.T.J. and Gascon, J. (2000) Quinolone resistance in enterotoxigenic Escherichia coli causing diarrhea in travelers to India in comparison with other geographical areas. Antimicrob. Agents Chemother, 44(6): 1731-1733.

36. Olorunshola, I.D., Smith, S.I. and Coker, A.O. (2000) Prevalence of ehec o157: h7 in patients with diarrhoea in Lagos, Nigeria. APMIS, 108(11): 761-763.

37. Thakur, S. and Gray, G.C. (2019) The mandate for a global "one health" approach to antimicrobial resistance surveillance. Am. J. Trop. Med. Hyg., 100(2): 227-228. 


\section{Additional File 1}

Table-S1: Primer characteristics used in the multiplex polymerase chain reaction.

\begin{tabular}{|c|c|c|c|c|}
\hline Primers & Target gene & Sequence & Size (bp) & AT $\left({ }^{\circ} \mathrm{C}\right)$ \\
\hline LT & eltB & $\begin{array}{l}\text { 5-TCTCTATGTGCATACGGAGC-3 } \\
\text { 5-CCATACTGATTGCCGCAAT-3 }\end{array}$ & 322 & 56 \\
\hline ST & est $A$ & $\begin{array}{l}\text { 5-GCTAAACCAGTAGAGGTCTTCAAAA-3 } \\
\text { 5-CCCGGTACAGAGCAGGATTACAACA-3 }\end{array}$ & 147 & 56 \\
\hline VT1 & $v t 1$ & $\begin{array}{l}\text { 5-GAAGAGTCCGTGGGATTACG-3 } \\
\text { 5-AGCGATGCAGCTATTAATAA-3 }\end{array}$ & 130 & 56 \\
\hline VT2 & $v t 2$ & $\begin{array}{l}\text { 5-ACCGTTITCAGATITTGACACATA-3 } \\
\text { 5-TACACAGGAGCAGTTTCAGACAGT-3 }\end{array}$ & 298 & 53 \\
\hline Eae & eaeA & $\begin{array}{l}\text { 5-CACACGAATAAACTGACTAAAATG-3 } \\
\text { 5-AAAAACGCTGACCCGCACCTAAAT-3 }\end{array}$ & 376 & 54 \\
\hline SHIG & ial & $\begin{array}{l}\text { 5-CTGGTAGGTATGGTGAGG-3 } \\
\text { 5-CCAGGCCAACAATTATTTCC-3 }\end{array}$ & 320 & 53 \\
\hline bfpA & $b f p A$ & $\begin{array}{l}\text { 5-TTCTTGGTGCTTGCGTGTCTIT-3 } \\
\text { 5-TTITGTTTGTTGTATCTTTGTAA-3 }\end{array}$ & 367 & 56 \\
\hline EA & $p C V D$ & $\begin{array}{l}\text { 5-CTGGCGAAAGACTGTATCAT-3 } \\
\text { 5-CAATGTATAGAAATCCGCTGTT-3 }\end{array}$ & 630 & 53 \\
\hline
\end{tabular}

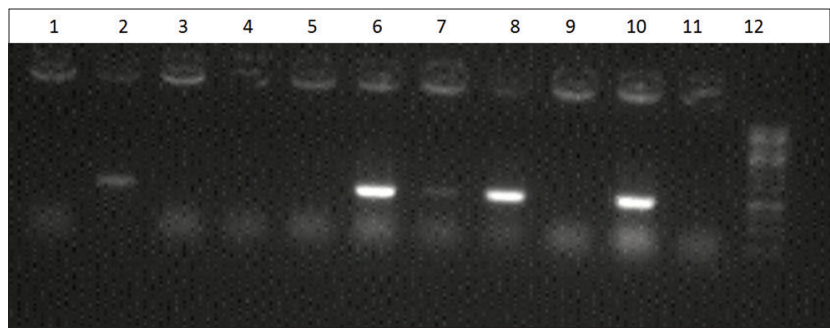

Figure-S1: Representative of molecular identification of Escherichia coli strains using uspA gene primers. Lines 2, $6,7,8$, and 10 show the presence of uspA genes in the isolates. Line 12 is DNA ladder.

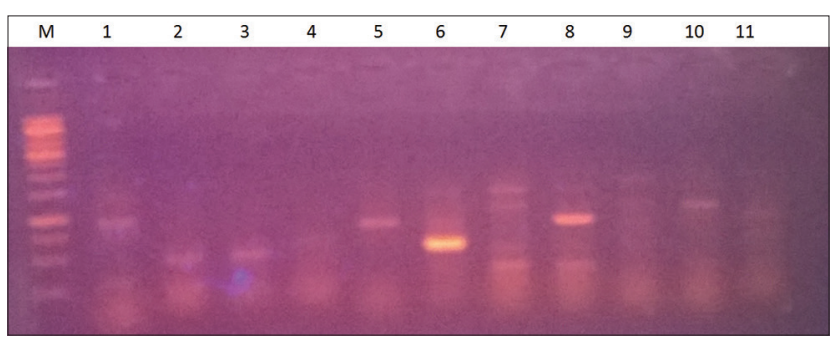

Figure-S2: Representative of multiplex polymerase chain reaction in identification of enterotoxigenic Escherichia coli and enteroaggregative $E$. coli using eltB/elt $A$ and $p C V D$ primers, respectively, Lane $M$, marker (1-kb DNA ladder); Lane 1-11, E. coli isolates.

$* * * * * * * *$ 